\title{
Morfometria de oocistos de Eimeria em bezerras segundo a faixa etária e a intensidade de infecção, Município de Piraí, RJ
}

\author{
Morphometry of "Eimeria" oocysts in heifer calves according to age group and \\ intensity of infection, municipality of Pirai, $R J$
}

\author{
VIDAL, Letícia Gabriela Poblete ${ }^{1 *}$; FAGUNDES, Thais Ferreira ${ }^{1}$; PANTOJA, Camila \\ Santos $^{1}$; MENEZES, Rita de Cássia Alves Alcantara de ${ }^{2}$
}

\author{
${ }^{1}$ Universidade Federal Rural do Rio de Janeiro,Instituto de Veterinária, Programa de Pós-Graduação em \\ Ciências Veterinárias, Seropédica, Rio de Janeiro, Brasil. \\ ${ }^{2}$ Universidade Federal Rural do Rio de Janeiro, Instituto de Veterinária, Departamento de Parasitologia \\ Animal, Seropédica, Rio de Janeiro, Brasil. \\ *Endereço para correspondência: let_vidal@yahoo.com.br.
}

\section{RESUMO}

Objetivou-se avaliar a variação média no tamanho dos oocistos de Eimeria, segundo as categorias das variáveis: faixa etária das bezerras (zero a 100 e 101 a 180 dias de idade) e intensidade de infecção bem como correlacionar as espécies do gênero Eimeria, utilizando técnicas de morfometria. De agosto/2010 a junho/2011, no Município de Piraí, RJ, foram coletadas 1512 amostras de fezes de 57 bezerras, sendo 955 enquanto estavam na faixa etária de 0 a 100 dias e 557 já com 101 a 180 dias. Com base nas características morfológicas e morfométricas dos oocistos esporulados, identificaram-se nove espécies nas seguintes porcentagens: $\quad E$. ellipsoidalis $(39,7 \%), \quad E$. alabamensis $(18,4 \%)$, E. bovis $(12,1 \%)$, E. zuernii $(11,4 \%), \quad$ E. subspherica $(7,3 \%)$, E. cylindrica $(6,0 \%)$, E. auburnensis $(3,6 \%)$, E. wyomingensis $(1,0 \%)$ e $E$. canadensis $(0,6 \%)$. As bezerras de até 100 dias apresentaram maior intensidade de infecção e, nas duas faixas etárias houve menos amostras classificadas como de alta intensidade. Pode-se observar que não houve correlação $(\mathrm{p}>0,05)$ entre as técnicas de medidas aplicadas, mostrando que uma independe da outra. Com base na análise dos oocistos da espécie E. ellipsoidalis, pode-se observar que a idade dos animais influenciou na morfometria dos oocistos em ambos os recursos de medida. Entretanto, não houve diferença significativa $(p>0,05)$ na morfometria dos oocistos de acordo com a intensidade de infecção.

Palavras-chave: bovinos, estudo morfométrico, prevalência de coccídio

\section{SUMMARY}

The aim of this study was evaluate the average variation in the size of oocysts of Eimeria, recovered from heifer calves, using the following variables: age of the calves (0-100 and 101-180 days of age) and intensity of infection. In addition two measurements techniques were employed to correlate the morphometry of Eimeria species. A total of 1512 fecal samples were collected from 57 heifer calves in the municipality of Pirai, Rio de Janeiro, during the period August/2010 to June/2011, with 955 collected during the period 0100 days and 557 from calves between 101-180 days old. Nine species of Eimeria were identified based on morphological and morphometric characteristics of oocysts and were present at the following percentages: E. ellipsoidalis (39.7\%), E. alabamensis (18.4\%), E. bovis (12.1\%), E. zuernii $(11.4 \%), \quad$ E. subspherica $(7.3 \%)$, E. cylindrica (6.0\%), E. auburnensis $(3.6 \%)$, E. wyomingensis $(1.0 \%)$ and E. canadensis $(0.6 \%)$. Calves up to 100 days old showed higher intensities of infection (\%). Yet, in both age groups high intensity infection was the least frequently observed category of infection. Statistical analysis did not reveal any correlation $(p>0.05)$ between the two measurement techniques, indicating that they were equal effective. In the specific case of the oocysts of E. ellipsoidalis, it was observed that the age of the animals influenced oocyst morphometry as analyzed using either measurement techniques. However, no significant difference $(p>0.05)$ in the morphometry of oocysts was observed in relation to the intensity of infection.

Keywords: cattle, coccidial prevalence, morphometric analisys 


\section{INTRODUÇ̃̃O}

A morfologia dos oocistos tende a apresentar aspectos que podem ser descritos de maneira qualitativa $\mathrm{e}$ quantitativa, com grande variação $\mathrm{e}$ combinação de características específicas (CARVALHO FILHO et al., 2004; HASSUM et al., 2007; LONG \& JOYNER, 1984). Uma característica morfológica importante no auxílio da diferenciação das espécies do gênero Eimeria é o índice morfométrico, que consiste na divisão dos diâmetros maior pelo menor. O tamanho do oocisto pode ser variável, mas seu índice morfométrico demonstra uma tendência retilínea que reflete a forma do oocisto, sendo mais precisa para comparação entre espécies do que a média das dimensões, como também na variação intraespecífica (LONG \& JOYNER, 1984).

Em bezerros a infecção ocorre principalmente entre três semanas e seis meses de idade. $\mathrm{O}$ aumento das taxas de morbidade e de mortalidade e a baixa eficiência alimentar dos animais (REHMAN et al., 2011) são reflexo da infecção. Os bezerros são mais sensíveis à infecção devido à grande diversidade de fatores predisponentes aos quais estão expostos, como falhas na ingestão do colostro, dificuldades de adaptação ao ambiente de confinamento, além da falta de uma resposta imune específica para proteção contra a doença (SÁNCHEZ et al., 2008).

A intensidade de infecção é maior na estação chuvosa em relação à seca (REHMAN et al., 2011). Os animais alimentados diretamente no solo ou pastagem têm mais chances de ingestão de oocistos em comparação com aqueles alimentados em calhas de alimentação (ABEBE et al., 2008).

$\mathrm{Na}$ literatura há poucos relatos acerca da morfometria de eimeriídeos relacionada à intensidade de infecção, traduzida pela quantidade de oocistos por grama de fezes (OoPG), em bezerros. Tal fato assume relevância, ao considerar que os oocistos são utilizados para o diagnóstico e avaliação do potencial patogênico das espécies envolvidas na infecção. Assim, o presente trabalho teve como objetivos identificar as espécies do gênero Eimeria que infectam bezerras, avaliar a variação no tamanho dos oocistos nas variáveis faixa etária (de zero a 100 e de 101 a 180 dias de idade) e intensidade de infeção, e correlacionar os resultados de morfometria obtidos por meio de ocular micrométrica com a do sistema de digitalização de imagem.

\section{MATERIAL E MÉTODOS}

Foram utilizadas bezerras mestiças, com graus variados de sangue da raça holandesa preta e branca, de duas faixas etárias (do nascimento até 100 dias e de 101 a 180 dias de idade) e nascidas no período de agosto de 2010 a janeiro de 2011. De modo que havia a introdução gradual de animais no estudo, assim como após 180 dias de vida, deixavam de participar da pesquisa, o que conferiu uma flutuação no número de animais por faixa etária. As coletas foram encerradas em junho de 2011 e no período participaram do estudo um total de 57 bezerras.

Na propriedade localizada no Município de Piraí, estado do Rio de Janeiro, havia um banco de colostro de boa qualidade e congelado, selecionado após o teste usando colostrômetro. As bezerras após o nascimento eram separadas imediatamente de suas mães, e, em mamadeira, o tratador fornecia seis litros de tal colostro.

As instalações do criatório consistiam em abrigos individuais em piquetes para 
as fêmeas até 100 dias de idade. As bezerras de 101 a 180 dias eram acomodadas em piquetes com espaço coberto (bezerreiro coletivo) onde havia cochos de alvenaria.

As coletas de fezes, direto da ampola retal, foram realizadas semanalmente. Em seguida eram acondicionadas em sacos plásticos e mantidas sob refrigeração até o momento de serem examinadas. Ao final do estudo, o total de amostras coletado foi 1.512.

Parte das fezes de cada bezerra foi processada pela técnica de centrífugoflutuação em solução saturada de açúcar $(\mathrm{d}=1,30)$ de acordo com o descrito por Menezes \& Lopes (1995), para então obter o número de oocistos por grama de fezes (OoPG) e assim classificar a intensidade de infecção em moderada e alta. Ueno \& Gonçalves (1998) com base na eliminação de ovos por grama de fezes (OPG) apresentaram graus de infecção mista de bovinos por nematóides em moderada (200-700) e pesada (700). Assim, considerando que as infecções por espécies de Eimeria também são multiespecíficas e apresentam as mesmas variações citadas por Ueno \& Gonçalves (1998), foi feita a extrapolação dos parâmetros desses autores para classificar, neste estudo, a intensidade de infecção em moderada $(5-700)$ e alta $(>700)$.

Considerando as categorias das variáveis (faixa etária e intensidade de infecção), à outra parte da amostra fecal, foi acrescentada solução de dicromato de potássio a 2,5\% na proporção de (1:2) para que ocorresse a esporulação em temperatura ambiente para posterior identificação percentual das espécies de Eimeria, segundo os critérios de Levine (1985) e Eckert et al. (1995). Em seguida foram medidos 100 oocistos de cada espécie, dentre aquelas mais prevalecentes, cujos oocistos eram mais abundantes, para cada categoria das variáveis. As medidas foram feitas a partir de oocistos provenientes de uma mesma amostra, nas duas técnicas. Para esse fim foi utilizada uma ocular micrométrica com o aumento de $15 \mathrm{X}$ (PZO) acoplada ao microscópio óptico (WILD, modelo M11) e também por meio de um sistema de digitalização de imagem, com uso do $\mathrm{Cell}^{\mathrm{R}} \& \mathrm{Cell}^{\mathrm{R}}$ Imaging Software for Life Science Microscopy e câmera fotográfica (UC30) adaptada a um microscópio binocular Olympus BX51.

De cada oocisto foram medidos os diâmetros maior e menor, a largura da parede e calculado o índice morfométrico (IM). De um esporocisto por oocisto, foram medidos os diâmetros maior e menor e também calculado seu IM.

$\mathrm{Na}$ análise estatística dos resultados, foram aplicados os testes de Spearman para avaliar se houve diferenças entre as análises do sistema digital de imagens em relação às medidas realizadas com o uso da ocular micrométrica. Para verificar se a idade dos animais e a intensidade de infecção influenciaram no tamanho do oocisto, utilizou-se o teste de Mann-Whitney (R FOUNDATION FOR STATISTICAL COMPUTING, 2011).

\section{RESULTADOS E DISCUSSÃO}

Com base nas características morfológicas e morfométricas dos oocistos esporulados identificaram-se nove espécies de Eimeria nas bezerras estudadas: $E$. alabamensis, $E$. auburnensis, E. bovis, E. canadensis, E. cylindrica, E. ellipsoidalis, , E. subspherica, E. wyomingensis, $E$. zuernii. As espécies mais prevalecentes foram E. ellipsoidalis, E. alabamensis e E. bovis (Figura 1). 


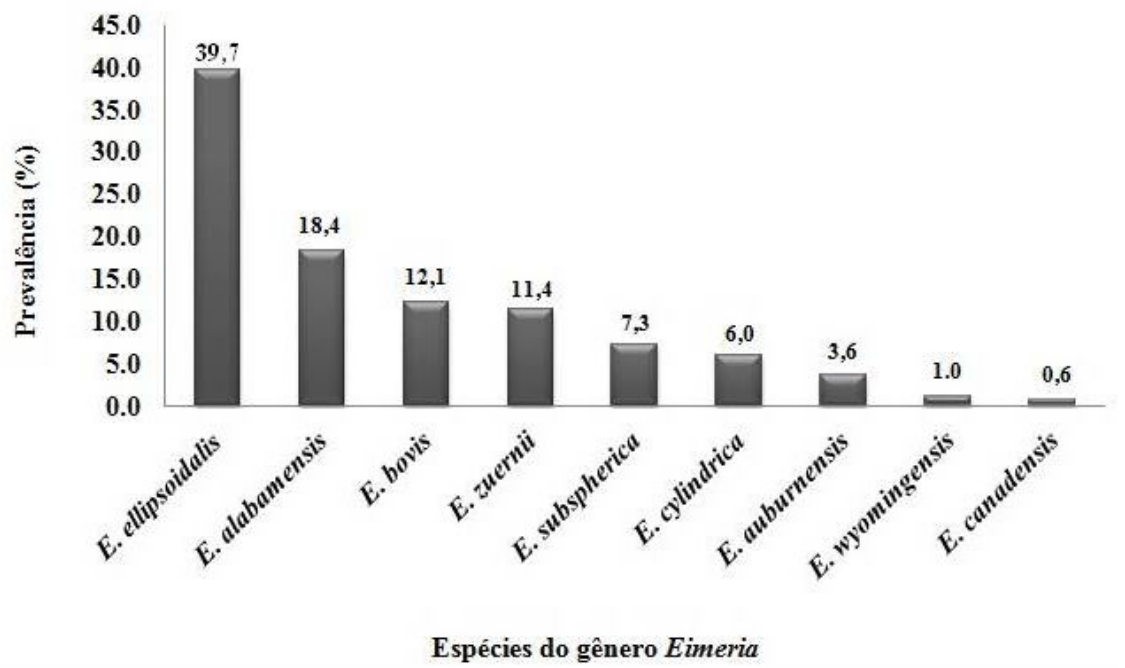

Figura 1. Prevalência das espécies de Eimeria identificadas infectando bezerras das duas faixas etárias, Município Piraí, RJ

Observou-se que a infecção é multiespecífica, como observado neste estudo e nas pesquisas Lucas et al. (2007) e de Sánchez et al. (2008), que também observaram a infecção em bovinos jovens. Entretanto, a diversidade e o número de espécies presentes em diferentes estudos é variável, como se pode verificar pelos resultados de Bruhn et al. (2011) e de Koutny et al. (2011), e também pelo observado na Alemanha por Von Samson-Himmelstjerna et al. (2006) que identificaram apenas três espécies (E. alabamensis, E. bovis e E. zuernii), e no Brasil por Bruhn et al. (2012) que identificaram dez espécies, sendo $E$. bovis $(37,6 \%)$ e E. zuernii $(17,9 \%)$ as mais frequentes. Mesmo que as espécies mais prevalecentes tenham sido as mesmas como neste estudo ( $E$. ellipsoidalis, E. alabamensis e E. bovis) e nos de Sánchez et al. (2008) na Argentina, Rehman et al. (2011) no Paquistão e de Koutny et al. (2011) na Áustria, a prevalência pode ser bastante diferente.

O clima e a resposta imune do hospedeiro podem influenciar a diversidade (LUCAS et al., 2007) e essas variações podem ser atribuídas às diferentes regiões geográficas onde os trabalhos foram realizados, ao tipo de manejo adotado e também aos fatores inerentes aos hospedeiros, como a faixa etária e raça dos animais estudados. Ainda segundo Lucas et al. (2007) as interações entre hospedeiro, parasita e ambiente são complexas, mas a composição das espécies de Eimeria podem se manter estáveis em bezerros, com pequenas e graduais alterações ocorrendo nas semanas seguintes ao desmame.

Embora E. ellipsoidalis tenha sido a espécie mais prevalecente, na faixa etária de zero a 100 dias com alta infecção, E. bovis foi mais frequente nas infecções moderadas (Tabela 1). Tal resultado foi encontrado também por Almeida et al. (2011) no sudeste da Bahia, onde E. bovis foi a espécie mais prevalecente, sobretudo em bezerros com menos de um ano de idade. Já $E$. alabamensis apresentou frequência similar à de E. ellipsoidalis nas bezerras de 101-180 com intensidade de infecção alta. 
Tabela 1.Frequência (\%) das espécies do gênero Eimeria encontradas em bezerras de acordo com a faixa etária (0-100 e de 101- 180 dias) e a intensidade de infecção, no Município de Piraí, RJ

\begin{tabular}{lcccc}
\hline \multirow{2}{*}{ Espécies } & \multicolumn{3}{c}{ Moderada } & \multicolumn{2}{c}{ Alta } \\
\cline { 2 - 5 } & $0-100$ & $101-180$ & $0-100$ & $101-180$ \\
\hline E. subspherica & 7,1 & 9,0 & 3,0 & 10,0 \\
E. zuernii & 15,0 & 3,0 & 12,0 & 15,5 \\
E. ellipsoidalis & 29,0 & 56,0 & 40,3 & 33,4 \\
E. cylindrica & 5,0 & 0,6 & 13,7 & 4,7 \\
E. alabamensis & 6,6 & 15,3 & 19,0 & 32,5 \\
E. bovis & 31,5 & 5,7 & 9,2 & 2,7 \\
E. canadensis & 2,0 & 0 & 0,15 & 0,4 \\
E.wyomingensis & 1,1 & 0 & 2,15 & 0,8 \\
E. auburnensis & 2,7 & 11,0 & 0,5 & 0 \\
\hline
\end{tabular}

No presente estudo a primeira eliminação de oocistos, ocorreu aos seis dias de idade com média 22,39 $\pm 7,59$ $($ moda $=25)$. Noronha Junior et al. (2009) obtiveram em búfalos o mesmo resultado para o primeiro dia de encontro da infecção. Embora Sánchez et al. (2008) não relatem a primeira observação de oocistos, verificaram que ocorre aumento de OoPG entre 21 e 35 dias de idade. Em São Paulo Barbosa et al., (1992) encontraram 58\% de bezerros búfalos infectados com espécies do gênero Eimeria após 21 dias de idade. Estes autores sugeriram que os bezerros podem ser infectados logo após o nascimento, possivelmente durante a amamentação, através da ingestão de oocistos aderidos ao úbere das vacas. Neste estudo a higiene do local de alojamento das bezerras até 100 dias era negligenciada. E a presença eventual de animais adultos com consequente eliminação de fezes contaminando o ambiente foi a provável fonte de infecção para as bezerras, já que a limpeza e desinfecção das instalações são fundamentais para $o$ controle da coccidiose em bezerros, como destacado por Bangoura et al. (2012).

Considerando que as mesmas bezerras foram acompanhadas de zero a 180 dias de idade, os animais com até 100 dias apresentaram maior percentual de infecção (Tabela 2), corroborando os resultados de Cicek et al. (2007) na Turquia, Rehman et al., (2011) no Paquistão, e de Almeida et al., (2011) no Brasil, onde os bovinos jovens apresentaram maior intensidade de infecção em relação aos mais velhos. No entanto, somente $114(12,96 \%)$ do total de 879 amostras positivas foram classificadas como apresentando intensidade alta, em ambas as faixas etárias.

Tabela 2. Intensidade de infecção por Eimeria, de acordo com a faixa etária das bezerras, no Município de Piraí

\begin{tabular}{lcc}
\hline \multirow{2}{*}{ Intensidade de Infecção } & \multicolumn{2}{c}{ Faixa Etária (dias) } \\
\cline { 2 - 3 } & $0-100$ & $101-180$ \\
\hline Negativa & $446(29,5 \%)$ & $187(12,3 \%)$ \\
Moderada & $433(28,6 \%)$ & $332(21,9 \%)$ \\
Alta & $76(5 \%)$ & $38(2,7 \%)$ \\
\hline
\end{tabular}


O estudo morfométrico das cinco espécies mais prevalecentes e com maior abundância de oocistos (Figura 2) foi realizado para a comparação entre os métodos de medidas.

Através das médias das medidas obtidas pelos dois métodos de medida dos oocistos e esporocistos encontrados (Tabelas 3, 4, 5 e 6), considerando a amplitude de variação, assim como a morfologia, pode-se observar que $E$. ellipsoidalis apresentou oocisto elipsoidal ou muito pouco ovoide. A parede do oocisto apresentou-se fina, com ausência de micrópila. As medidas dos oocistos variaram de $22-26 \mu \mathrm{m}$ (diâmetro maior) por $14-17 \mu \mathrm{m}$ (diâmetro menor) e não apresentaram corpo residual. Os esporocistos apresentaram 11 - $15 \mu \mathrm{m}$ (diâmetro maior) por 4 - $6 \mu \mathrm{m}$ (diâmetro menor) com presença de resíduo no esporocisto. Os esporozoitos eram alongados com um glóbulo na parte mais larga e um próximo ao meio Eimeria zuernii apresentou oocistos subsféricos ou ovoides e não apresentou micrópila. Os

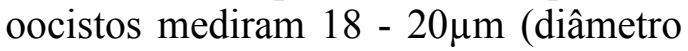
maior) por 15 - $17 \mu \mathrm{m}$ (diâmetro menor) e não apresentaram resíduo de oocisto. Os esporocistos eram alongados $\mathrm{e}$ ovoides, com 10 - $12 \mu \mathrm{m}$ (diâmetro maior) por $5-6 \mu \mathrm{m}$ (diâmetro menor). Eimeria alabamensis apresentou oocisto ovoide, com ausência de micrópila e tamanho variando entre $19-23 \mu \mathrm{m}$ (diâmetro maior) por $14-17 \mu \mathrm{m}$ (diâmetro menor). Os esporocistos eram alongados, sem resíduo e variando de $10-13 \mu \mathrm{m}$ (diâmetro maior) por $4-6$ $\mu \mathrm{m}$ (diâmetro menor). De acordo com Levine (1985), esta espécie se localiza primariamente no intestino delgado, mas também no ceco e cólon em infecções severas, mas é essencialmente não patogênica em condições de campo. Eimeria bovis apresentou oocistos subsféricos ou ovoides e não apresentou micrópila. Os oocistos mediram 28 $32 \mu \mathrm{m}$ (diâmetro maior) por $21-24 \mu \mathrm{m}$ (diâmetro menor) e não apresentaram resíduo de oocisto. Os esporocistos eram alongados e ovoides, com 14 $18 \mu \mathrm{m}$ (diâmetro maior) por $6-14 \mathrm{~mm}$ (diâmetro menor).

Eimeria subspherica apresentou oocistos subsférico ou ovoide e não apresentou micrópila. Os oocistos mediram 14 - $17 \mu \mathrm{m}$ (diâmetro maior) por $13-16 \mu \mathrm{m}$ (diâmetro menor) e não apresentaram resíduo de oocistos, Os esporocistos eram alongados e ovoides, com $6-8 \mu \mathrm{m}$ (diâmetro maior) por 4$5 \mu \mathrm{m}$ (diâmetro menor).

Com base nos resultados morfométricos obtidos não houve correlação $(p>0,05)$ entre as técnicas aplicadas, mostrando que uma independe da outra. No entanto, as médias das medidas mostraram-se dentro dos parâmetros de referência adotados e próximos aos valores médios do padrão conforme as descrições de Eckert et al. (1995), comprovando que ambas são válidas.

Para efeito de obtenção de resultado, não faz diferença a técnica escolhida, a não ser quando considerada a praticidade e a rapidez de execução no sistema de digitalização de imagens. Com isso, pode ser considerada mais adequada como método de medida, inclusive porque a interferência do pesquisador é menor.

Após análise dos oocistos de $E$. ellipsoidalis, pode-se observar com uso do teste de Mann-Whitney, que a idade dos animais influenciou na forma dos oocistos em ambas as técnicas de medidas. Previamente Long \& Joyner (1984) assinalaram que apesar do pleomorfismo existente nos oocistos o seu índice morfométrico se manteria constante.

O pleomorfismo observado nos oocistos de E. ellipsoidalis e relacionado à idade das bezerras, pode ter sido em 
consequência do fator populacional, já que existe uma especificidade do coccídio ao sítio de infecção no intestino e que esta espécie foi a mais prevalecente e abundante, aliado ao fato da imaturidade do sistema imune do hospedeiro.

Não houve diferença significativa ( $>0,05)$ na morfometria dos oocistos de acordo com a intensidade de infecção. Embora possam existir variações individuais intraespecíficas, principalmente em infecções intensas e no final do período de patência, quando aumenta o número de oocistos deformados. Também, Levine (1985) afirma que o tamanho do oocisto não é constante, entretanto as dimensões dos mesmos são frequentes e consideradas como caracteres específicos.

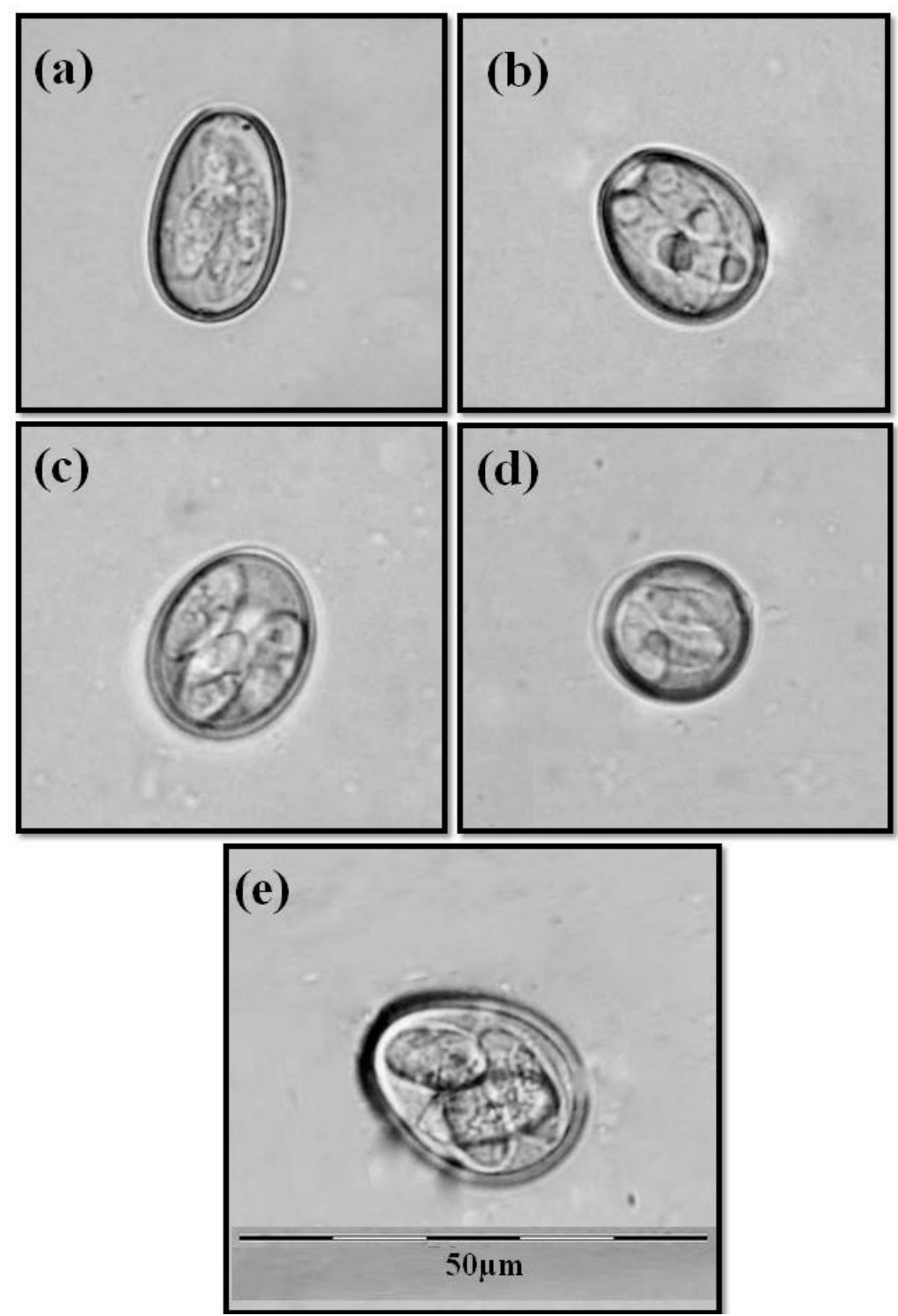

Figura 2. Oocistos esporulados das cinco espécies do gênero Eimeria mais prevalecentes em bezerras de zero a 180 dias de vida no Município de Piraí, RJ: (a) $E$. ellipsoidalis; (b) E. alabamensis; (c) E. zuernii; (d) E. subspherica; (e) E. bovis (solução saturada de açúcar. Escala $50 \mu \mathrm{m})$ 
Tabela 3. Morfometria dos oocistos esporulados, obtida com uso da ocular micrométrica,das espécies do gênero Eimeria de bezerras de 0100 dias de idade e, de acordo com a intensidade de infecção (moderada e alta), no Município de Piraí, RJ

\begin{tabular}{|c|c|c|c|c|c|c|c|}
\hline \multicolumn{8}{|c|}{ Moderada } \\
\hline \multirow{3}{*}{ Espécie } & \multicolumn{3}{|c|}{ Oocisto $^{a}$} & \multicolumn{4}{|c|}{ Esporocisto $^{a}$} \\
\hline & \multicolumn{2}{|c|}{ Diâmetro } & \multirow{2}{*}{ Parede } & \multirow{2}{*}{ I.M. } & \multicolumn{2}{|c|}{ Diâmetros } & \multirow{2}{*}{ I.M. $^{\mathrm{b}}$} \\
\hline & Maior & Menor & & & Maior & Menor & \\
\hline E. bovis & $28,7 \pm 1,53$ & $21,7 \pm 1,03$ & $1,6 \pm 0,16$ & $1,3 \pm 0,06$ & $14,9 \pm 1,17$ & $6,5 \pm 0,59$ & $2,3 \pm 0,28$ \\
\hline E. ellipsoidalis & $25,6 \pm 0,99$ & $17,0 \pm 0,98$ & $1,3 \pm 0,14$ & $1,5 \pm 0,08$ & $13,7 \pm 0,94$ & $5,7 \pm 0,49$ & $2,4 \pm 0,22$ \\
\hline E. alabamensis & - & - & - & - & - & - & - \\
\hline E. subspherica & - & - & - & - & - & - & - \\
\hline E. zuernii & - & - & - & - & - & - & - \\
\hline \multicolumn{8}{|l|}{ Alta } \\
\hline E. bovis & $31,4 \pm 2,15$ & $23,5 \pm 1,22$ & $2,0 \pm 0,35$ & $1,3 \pm 0,01$ & $18,2 \pm 1,56$ & $7,7 \pm 0,63$ & $2,3 \pm 0,24$ \\
\hline E. ellipsoidalis & $23,5 \pm 1,5$ & $16,0 \pm 1,01$ & $1,4 \pm 0,18$ & $1,4 \pm 0,01$ & $13,1 \pm 1,12$ & $5,4 \pm 0,68$ & $2,4 \pm 0,37$ \\
\hline E. alabamensis & - & - & - & - & - & - & - \\
\hline E. subspherica & - & - & - & - & - & - & - \\
\hline E.zuernii & $19,4 \pm 0,96$ & $17,0 \pm 1,19$ & $1,3 \pm 0,24$ & $1,14 \pm 0,06$ & $11,1 \pm 1,03$ & $5,6 \pm 0,64$ & $2,0 \pm 0,26$ \\
\hline
\end{tabular}

${ }^{\mathrm{a}}$ Média \pm desvio padrão; ${ }^{\mathrm{b}} \mathrm{IM}=$ Índice Morfométrico.

E. alabamensis, E. subspherica e E. zuernii não tiveram oocistos suficientes para mensuração na infecção moderada.

E. alabamensis e E. subspherica não tiveram oocistos suficientes para mensuração na infecção alta. 
Tabela 4. Morfometria dos oocistos esporulados, obtida com uso da ocular micrométrica, das espécies do gênero Eimeria de bezerras de 101-180 dias de idade e, de acordo com a intensidade de infecção (moderada e alta), no Município de Piraí, RJ

\begin{tabular}{|c|c|c|c|c|c|c|c|}
\hline \multirow{4}{*}{ Espécie } & \multicolumn{6}{|c|}{ Moderada } & \\
\hline & \multicolumn{2}{|c|}{ Oocisto $^{a}$} & & \multicolumn{4}{|c|}{ Esporocisto $^{\mathbf{a}}$} \\
\hline & \multicolumn{2}{|c|}{ Diâmetro } & \multirow{2}{*}{ Parede } & \multirow{2}{*}{ I.M. } & \multicolumn{2}{|c|}{ Diâmetros } & \multirow{2}{*}{ I.M. $^{\mathrm{b}}$} \\
\hline & Maior & Menor & & & Maior & Menor & \\
\hline E. ellipsoidalis & $25,3 \pm 1,69$ & $17,0 \pm 1,13$ & $1,2 \pm 0,17$ & $1,5 \pm 0,09$ & $13,9 \pm 1,12$ & $5,7 \pm 0,45$ & $2,4 \pm 0,224$ \\
\hline \multicolumn{8}{|c|}{ Alta } \\
\hline E. ellipsoidalis & $26,5 \pm 1,76$ & $16,2 \pm 1,94$ & $1,3 \pm 0,18$ & $1,6 \pm 0,13$ & $13,4 \pm 0,9$ & $5,2 \pm 0,5$ & $2,6 \pm 0,28$ \\
\hline E. alabamensis & $22,1 \pm 1,58$ & $16,1 \pm 0,87$ & $1,3 \pm 0,18$ & $1,4 \pm 0,09$ & $12,6 \pm 0,92$ & $5,1 \pm 0,4$ & $2,5 \pm 0,25$ \\
\hline E. subspherica & $16,8 \pm 0,89$ & $15,4 \pm 0,89$ & $1,3 \pm 0,21$ & $1,1 \pm 0,04$ & $7,5 \pm 1,34$ & $4,5 \pm 0,39$ & $1,7 \pm 0,28$ \\
\hline E.zuernii & $18,9 \pm 2,06$ & $16,6 \pm 1,3$ & $1,3 \pm 0,06$ & $1,2 \pm 0,07$ & $13,4 \pm 1,03$ & $5,0 \pm 0,51$ & $2,2 \pm 0,29$ \\
\hline
\end{tabular}

${ }^{\mathrm{a}}$ Média \pm desvio padrão; ${ }^{\mathrm{b}} \mathrm{IM}=$ Índice Morfométrico.

E. bovis, E. alabamensis, E. subspherica e E. zuernii não tiveram oocistos suficientes para mensuração na infecção moderada.

E. bovis não teve oocistos suficientes para mensuração na infecção alta.

Tabela 5. Morfometria dos oocistos esporulados, obtida com o uso do sistema digital de imagem, das espécies do gênero Eimeria de bezerras de 0-100 dias de idade e, de acordo com a intensidade de infecção (moderada e alta), no Município de Piraí, RJ

\begin{tabular}{|c|c|c|c|c|c|c|c|}
\hline \multirow{4}{*}{ Espécie } & \multicolumn{6}{|c|}{ Moderada } & \\
\hline & \multicolumn{3}{|c|}{ Oocisto $^{a}$} & \multicolumn{4}{|c|}{ Esporocisto $^{\mathrm{a}}$} \\
\hline & \multicolumn{2}{|c|}{ Diâmetro } & \multirow{2}{*}{ Parede } & \multirow{2}{*}{ I.M. } & \multicolumn{2}{|c|}{ Diâmetros } & \multirow{2}{*}{ I.M. ${ }^{b}$} \\
\hline & Maior & Menor & & & Maior & Menor & \\
\hline E. bovis & $31,2 \pm 1,72$ & $23,4 \pm 2,28$ & $1,9 \pm 0,24$ & $1,3 \pm 0,27$ & $16,5 \pm 1,83$ & $7,1 \pm 0,87$ & $2,3 \pm 0,52$ \\
\hline E. ellipsoidalis & $22,9 \pm 1,71$ & $15,5 \pm 1,85$ & $1,2 \pm 0,04$ & $1,5 \pm 0,45$ & $11,9 \pm 0,93$ & $4,8 \pm 0,54$ & $2,4 \pm 0,3$ \\
\hline \multicolumn{8}{|c|}{ Alta } \\
\hline E. bovis & $28,18 \pm 1,44$ & $21,8 \pm 0,92$ & $1,9 \pm 0,29$ & $1,3 \pm 0,08$ & $15,7 \pm 1,49$ & $14,7 \pm 0,75$ & $2,3 \pm 0,38$ \\
\hline E. ellipsoidalis & $25,1 \pm 1,66$ & $17,1 \pm 1,05$ & $1,4 \pm 0,25$ & $1,4 \pm 0,09$ & $14,5 \pm 1,44$ & $6,0 \pm 0,63$ & $2,4 \pm 0,37$ \\
\hline E.zuernii & $18,9 \pm 1,3$ & $15,7 \pm 1,34$ & $1,2 \pm 0,1$ & $1,2 \pm 0,08$ & $10,4 \pm 3,2$ & $5,3 \pm 0,53$ & $2,0 \pm 0,73$ \\
\hline
\end{tabular}

${ }^{\mathrm{a}}$ Média \pm desvio padrão; ${ }^{\mathrm{b}} \mathrm{IM}=$ Índice Morfométrico.

E. alabamensis, E. subspherica e E. zuernii não tiveram oocistos suficientes para mensuração na infecção moderada.

E. alabamensis e E. subspherica não tiveram oocistos suficientes para mensuração na infecção alta. 
Tabela 6. Morfometria dos oocistos esporulados, obtida com o uso do sistema digital de imagem, das espécies do gênero Eimeria de bezerras de 101-180 dias de idade e, de acordo com a intensidade de infecção (moderada e alta), no Município de Piraí, RJ

\begin{tabular}{|c|c|c|c|c|c|c|c|}
\hline \multicolumn{8}{|c|}{ Moderada } \\
\hline \multirow{3}{*}{ Espécie } & \multicolumn{2}{|c|}{ Oocisto $^{a}$} & & \multicolumn{4}{|c|}{ Esporocisto $^{a}$} \\
\hline & \multicolumn{2}{|c|}{ Diâmetro } & \multirow{2}{*}{ Parede } & \multirow{2}{*}{ I.M. } & \multicolumn{2}{|c|}{ Diâmetros } & \multirow{2}{*}{ I.M. ${ }^{b}$} \\
\hline & Maior & Menor & & & Maior & Menor & \\
\hline E. ellipsoidalis & $23,4 \pm 1,36$ & $15,9 \pm 0,91$ & $1,3 \pm 0,09$ & $1,5 \pm 0,1$ & $12,4 \pm 1,02$ & $5,1 \pm 0,51$ & $2,5 \pm 0,3$ \\
\hline \multicolumn{8}{|c|}{ Alta } \\
\hline E. ellipsoidalis & $24,0 \pm 1,94$ & $14,9 \pm 1,0$ & $1,2 \pm 0.07$ & $1,6 \pm 0,14$ & $11,7 \pm 1,48$ & $4,8 \pm 0,54$ & $2,4 \pm 0,39$ \\
\hline E. alabamensis & $19,4 \pm 1,8$ & $14,5 \pm 0,93$ & $1,3 \pm 0,1$ & $1,3 \pm 0,13$ & $10,6 \pm 0,82$ & $4,0 \pm 0,55$ & $2,3 \pm 0,3$ \\
\hline E. subspherica & $14,3 \pm 1,27$ & $13,3 \pm 1,34$ & $1,1 \pm 0,11$ & $1,1 \pm 0,06$ & $6,9 \pm 1,27$ & $4,0 \pm 0,55$ & $1,8 \pm 0,39$ \\
\hline E.zuernii & $18,9 \pm 1,3$ & $15,7 \pm 1,34$ & $1,2 \pm 0,1$ & $1,2 \pm 0,08$ & $10,4 \pm 0,99$ & $5,0 \pm 0,58$ & $2,1 \pm 0,24$ \\
\hline
\end{tabular}

${ }^{\mathrm{a}}$ Média \pm desvio padrão, ${ }^{\mathrm{b}} \mathrm{IM}=$ Índice Morfométrico.

E. bovis, E. alabamensis, E. subspherica e E. zuernii não tiveram oocistos suficientes para mensuração na infecção moderada.

E. bovis não teve oocistos suficientes para mensuração na infecção alta. 
Os resultados morfométricos encontrados por Noronha Junior et al. (2009) em um estudo realizado no Brasil, com bezerros búfalos até 133 dias de idade, foram diferentes do presente estudo, quando consideradas as seguintes espécies em comum: $E$. ellipsoidalis, E. subspherica e $E$. zuernii. A razão para as diferenças observadas pode ser atribuída a fatores inerentes ao hospedeiro, como estado imunológico, raça, idade, estado fisiológico, aqueles relacionados com o parasito como cepa e especificidade além dos ambientais, como umidade, temperatura e os atrelados ao sistema de manejo, que podem favorecer a esporulação dos oocistos de Eimerias spp. e a sua transmissão como comentado por Penzhorn et al. (1994).

Considerando que podem existir variações intraespecíficas, principalmente em infecções intensas, neste estudo a intensidade de infecção não interferiu na morfometria, porque a eliminação dos oocistos foi moderada, mesmo nas bezerras mais jovens.

\section{AGRADECIMENTOS}

À CAPES (Demanda Social) e à FAPERJ (E26/110.228/2010) pelo apoio financeiro.

\section{REFERÊNCIAS}

ABEBE, R.; WOSSENE, A.; KUMSA, B. Epidemiology of Eimeria infections in calves in Addis AbabaandDebreZeit dairy farms, Ethiopia. The

International Journal of Applied Research in Veterinary Medicine, v.6, n.1, p.24-30, 2008.
ALMEIDA, V.A.; MAGALHÃES, V.C.S.; MUNIZ NETA, E.S.; MUNHOZ, A.D. Frequency of species of genus Eimeria in naturally infected cattle in Southern Bahia, Northeast Brazil. Revista Brasileira de ParasitologiaVeterinária, v.20, n.1, p.78-81, 2011.

BANGOURA, B.; MUNDT, H.-C.; SCHMÃSCHKL, R.; WESTPHAL, B.; DAUGSECHIES, A. Prevalence of Eimeria bovis and Eimeria zuernii in German cattle herds and factors influencing oocyst excretion.

Parasitology Research, v.110, n.2, p.875-881, 2012.

BARBOSA, M.A.; BLASI, A.C.; OLIVEIRA, M.R. de; CORREA, F.M.A. Parasitismo natural de bufalinos em Botucatu, SP, Brasil - III. Dinâmica do parasitismo gastro-intestinal em vacas e suas crias. Memórias do Instituto Oswaldo Cruz, v.87, p.37-41, 1992. Supl. 1.

BRUHN, F.R.P.; LOPES, M.A.; DEMEU, F.A.; PERAZZA, C.A.; PEDROSA, M. F.; GUIMARÃES, A.M. Frequency of species of Eimeria in famales of the holstein-friesian breed at the post-weaning stage during autumn and winter. Revista Brasileira de Parasitologia Veterinária, v.20, n. 4, p.303-307, 2011.

BRUHN, F.R.P.; SILVA JÚNIOR, F.A.; CARVALHO, A.H.O.; ORLANDO, D.R.; ROCHA, C. M.B.M.; GUIMARÃES, A.M. Occurrences of Eimeria spp. and gastrointestinal nematodes in dairy calves in southern Minas Gerais, Brazil.

Revista Brasileira de Parasitologia Veterinária, v.21, n.2, p.171-175, 2012. 
CARVALHO FILHO, P.R.; MASSAD, F.V.; LOPES, C.W.G.; TEIXEIRA FILHO, W.L.; OLIVEIRA, F.C.R. Identificação e comparação de espécies do gênero Eimeria Schneider, 1875 (Apicomplexa: Eimeriidae) oriundas de suínos através de um algoritmo morfológico. Revista Brasileira de Ciências Veterinárias, v.11, n.3, p.156-159, 2004.

CICEK, H.; SEMVILI, F.; KOZAN, E.; KÖSE, M.; ESER, M.; DOGAN, N. Prevalence of coccidia in beef cattle in western Turkey. Parasitology

Research, v.101, n.5, p.1239-1243, 2007.

ECKERT, J.; BRAUN, R.; SHIRLEY, M.W.; COUDERT, P. (Ed.). Guidelines on techniques in coccidiosis research.

COST 89/820: Biotechnology, Luxembourg: Office for Official Publications of the European Communities, 1995. v.1, p.104-106.

FAYER, R. Epidemiology of protozoan infections: the coccidia. Veterinary Parasitology, v.6, n.1-3, p.75-103, 1980.

HASSUM, I.C.; VALLADARES, G.S.; MENEZES, R.C.A.A. Diferenciação das espécies de Eimeria parasitas de ovinos pelo uso da regressão linear e algoritmos morfológicos. Revista Brasileira de Parasitologia Veterinária, v.16, n.2, p.97-104, 2007.

KOUTNY, A.; JOACHIM, A.; TICHY, A.; BAUMGARTNER, W. Bovine Eimeria species in Austria.

Parasitology research, v. 10, n.5, p.1893-1901, 2011.

LEVINE, N.D. Veterinary

Protozoology. Ames: Iowa State University Press, 1985. 414p.
LONG, P.L.; JOYNER, L.P. Problems in the identification of species of Eimeria. Journal of Protozoology, v.31, n.4, p.535-541, 1984.

LUCAS, A.S.; SWECKER, W.S.; LINDSAY, D.S.; SCAGLIA, G.; ELVINGER, F.C.; ZAJAC, A.M. The effect of weaning method on coccidial infections in beef calves. Veterinary Parasitology, v.145, n.3-4, p.228-233, 2007.

MENEZES, R. C.A.A.; LOPES, C.W.G. Epizootiologia da Eimeria arloingi em caprinos na Microrregião Serrana Fluminense, Rio de Janeiro, Brasil. Revista Universidade Rural Série Ciências da Vida, v.17, n.2, p.512, 1995.

NORONHA JUNIOR, A.C.F.; STARKE-BUZETTI, W.A.; DUSZYNSKI, D.W. Eimeria spp. inbrazilianwaterbuffalo. The Journal of Parasitology, v.95, n.1, p.231-234, 2009.

PENZHORN, B.L.; ROGNLIE, M.C.; HALL, L.L.; KNNAP, S.E. Enteric coccidia of cashmere goats in southwestern Montana.Veterinary Parasitology, v.55, n.1, p.137-142, 1994.

REHMAN, T.U.; KHAN, M.N.; SAJID, M.S.; ABBAS, R.Z.; ARSHAD, M., IQBAL, Z.; IQBAL, A. Epidemiology of Eimeria and associated risk factors in cattle of district Toba Tek Singh, Pakistan. Parasitology Research, v.108, n.5, p.1171-1177, 2011.

R FOUNDATION FOR STATISTICAL COMPUTING. R: a language and environment for statistical computing: reference index. 2011. Disponível em: $<$ http://www.Rproject.org>. Acesso em: 15 set. 2011. 
SÁNCHEZ, R.O.; ROMERO, J.; FOUNROGE, R.D. Dynamics of Eimeria oocyst excretion in dairy calves in the Province of Buenos Aires (Argentina), during their first 2 months of age. Veterinary Parasitology, v.151, n.2-4, p.133-138, 2008.

UENO, H.; GONÇALVES, P.C. Manual para diagnóstico das helmintoses de ruminantes. 4.ed. Tóquio: Japan International Cooperation Agency, 1998. 143p.
VON SAMSON-HIMMELSTJERNA, G.; EPE, C.; WIRTHERLE, N.; VON DER, V. H.; WELZ, C.; RADELOFF, I.; BEENING, J.; CARR, D.; HELLMANN, K.; SCHNIEDER, T.; KRIEGER, K. Clinical and epimediological characteristics of Eimeria infections in first-year grazing cattle. Veterinary Parasitology, v.136, n.3-4, p.215-221, 2006.

Data de recebimento: 02/04/2013

Data de aprovação: 09/12/2013 